\title{
Gerinnungs-Selbstmanagement Flexible Einstellung, weniger Komplikationen
}

\author{
Die Sicherheit und Zuverlässigkeit \\ einer oraler Antikoagulation kann \\ durch ein Patienten-Selbstmanage- \\ ment verbessert werden. Gleichzeitig \\ gewinnen die Patienten Autonomie, \\ Flexibilität und Lebensqualität.
}

Die Qualität einer langfristigen Gerinnungshemmung mit Vitamin-K-Antagonisten (VKA) zur Schlaganfallprophylaxe wird durch zahlreiche Faktoren beeinflusst, wie Nahrung, enterale Vitamin-K-Synthese und Komedikation. Eine gute Einstellung der INR ist prognostisch relevant. Allerdings liegen selbst bei einer engen ärztlichen Betreuung nur 60\% der INR-Werte im Zielbereich, gab Prof. Markus Haas, Mannheim, zu bedenken.

Ein Gerinnungs-Selbstmanagement kann die Qualität der VKA-Therapie verbes- sern. 80\% der Patienten gelingt es so, die INR-Werte im therapeutischen Bereich zu halten. Metaanalytische Daten belegen, dass das Gerinnungs-Selbstmanagement gegenüber der reinen Gerinnungs-Selbstmessung überlegen ist. Die Thrombembolierate sinkt bei einem Gerinnungs-Selbstmanagement um 73\%, bei INR-Selbstkontrolle nur um 55\%. Auch die Sterblichkeit sinkt unter dem Selbstmanagement stärker als unter der alleinigen Selbsttestung ( -63 vs. $-39 \%$; Heneghan C et al. Lancet 2006;367:404-411). Außerdem werden die Flexibilität und die Unabhängigkeit der Patienten gefördert.

\section{Zuverlässig und kosteneffizient}

Studiendaten belegen, dass das GerinnungsSelbstmanagement unter oraler LangzeitAntikoagulation auch für die Kostenträger günstiger ist als die Praxismessung beim Hausarzt (Regier DA et al. CMAJ 2006;174: 1847-52).

Eine einfache, schnelle und zuverlässige Selbstmessung ermöglicht das mobile INRMessgerät CoaguChek ${ }^{\circledR}$ XS. Voraussetzung für ein Gerinnung-Selbstmanagement ist die erfolgreiche Teilnahme an einer entsprechenden Schulung, in der die Handhabung des Messgeräts, die Hintergründe der Blutgerinnung und die Bedeutung optimaler INR-Werte vermittelt werden. Laut Haas sind etwa 30-50\% der Patienten, die auf VKA eingestellt werden, schulbar.

(aam) II

II Pressegespräch anlässlich des 500000.

CoaguChek $^{\circledR}$ XS-Geräts, Mannheim,

2. September 2011 (Veranstalter: Roche Diagnostics)

\section{Neue Analysen der SHIFT-Studie Bessere Lebensqualität durch exklusive Herzfrequenzreduktion}

\begin{abstract}
Zwei Substudien der SHIFT-Studie mit kardiovaskulären Risikopatienten, die eine erhöhte Herzfrequenz hatten, bestätigen den symptomatischen und prognostischen Nutzen von Ivabradin.
\end{abstract}

In der kontrollierten SHIFT-Studie mit über 6500 Patienten hatte der $\mathrm{I}_{\mathrm{f}}$-Kanalblocker Ivabradin (Procoralan ${ }^{\oplus}$ ) zusätzlich zur optimalen medikamentösen Therapie mit ACEHemmern und Betablockern bei kardiovaskulären Risikopatienten (NYHA-Klasse II-IV) mit linksventrikulärer systolischer Dysfunktion (LVEF < 35\%) und erhöhter Herzfrequenz (> 70/min) zu einer signifikanten Reduktion des primären Endpunkts geführt. Dieser bestand aus kardiovaskulärem Tod und Hospitalisierung aufgrund einer Herzinsuffizienz.

Zusätzlich gestützt werden diese Befunde zur Senkung von kardiovaskulärer Morbidität und Mortalität durch die für Ivabradin in einer Substudie dokumentierte starke Reduktion des linksventrikulären Volumens bei Erhöhung der linksventrikulären

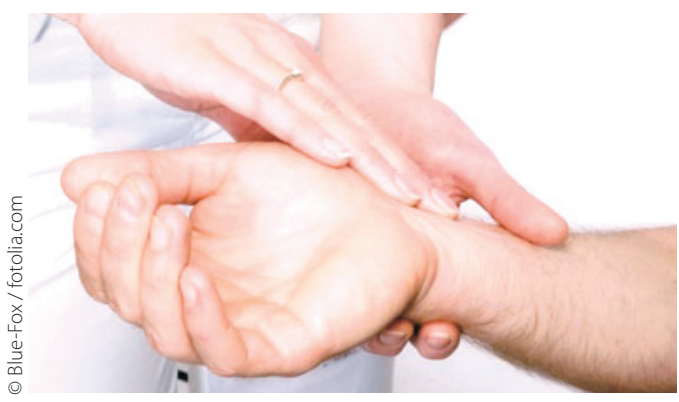

zu einer signifikanten Reduktion des LVESVI ( -7 vs. $-0,9 \mathrm{ml} / \mathrm{m}^{2}, \mathrm{p}<$ $0,001)$ und zur Steigerung der LVEF (+2,4 vs. $-0,1 \%, \mathrm{p}<0,001)$.

Dass Ivabradin zusätzlich zur Prognose auch die Symptome verbessert, belegt nach Borer eine zweite Substudie. Bei 1944 Patienten wurde zu Beginn, nach vier und zwölf Monaten die Lebensqualität mit dem Kansas City Cardiomyopathy Questionnaire (KCCQ) evaluiert. Nach einem Jahr wurde mit Mit einer Kombinationstherapie erreichen mehr Hypertoniker die geforderten Zielwerte.

Ejektionsfraktion (LVEF), so Prof. Jeffrey Borer, New York. Hierin waren 611 Patienten zu Studienbeginn und nach acht Monaten echokardiografisch erfasst worden. Primärer Endpunkt war die Veränderung des linksventrikulären endsystolischen Volumenindex (LVESVI), ein wichtiger sekundärer Endpunkt war die Veränderung der LVEF.

Trotz einer optimalen Pharmakotherapie führte die zusätzliche Herzfrequenzreduktion mit Ivabradin im Vergleich zu Placebo
Ivabradin eine im Vergleich zu Placebo signifikant bessere Lebensqualität ermittelt $(p=$ 0,018). Laut Borer zeigt sich die Reduktion kardiovaskulärer Endpunkte und Klinikeinweisungen mit Ivabradin in SHIFT somit auch in einer erhöhten Lebensqualität.

(loh)

II Satellitensymposium, ESC-Kongress, Paris, 30. August 2011 (Veranstalter: Servier) 